\title{
INTELLIGENT TWIN STEERING SYSTEM
}

\author{
Eng. Biagio Borretti*1, Eng. Nicola Musciagna ${ }^{1}$, Eng. Luca Riccò Ph.D. ${ }^{1}$, Eng. Andrea \\ Fornaciari ${ }^{2}$ \\ ${ }^{1}$ Dana Motion Systems Italia srl, Via Luciano Brevini 1/A, 42124 Reggio Emilia, Italy. \\ ${ }^{2}$ Engineering department Enzo Ferrari, University of Modena e Reggio-Emilia, Via P. Allegri 10, 41124 Modena. \\ *Corresponding author: Tel.: +39 0522505 870,+393389430 298; E-mail address: biagio.borretti@dana.com
}

\section{ABSTRACT}

This article describes the Dana intelligent Twin Steering System designed for mobile vehicles with two steering axles (i.e. with four steering wheels).

Current vehicles with four-wheel steering function are typically equipped with a hydraulic configuration where the two steering cylinders are connected in series. This type of connection highlights some limitations on available power, steering comfort and enabling advanced working functions.

Dana evolved this type of hydraulic configuration introducing the possibility to disconnect the rear axle from the front one. In this way the rear axle, through the electronic control of its valve block, allows to improve the steering system performance and introduces advanced steering functions compared to the conventional steering systems.

Keywords: Steering, 4 Wheels Steer, Control Strategy, Fuel Efficiency, Tyre Consumption, Comfort.

\section{INTRODUCTION}

Mobile heavy-duty vehicles equipped with two steering axles allow to switch between two steering wheels (2WS) functioning and four steering wheels (4WS) functioning by connecting the front axle hydraulic steering cylinder in series with the rear axle (Figure 1.1).

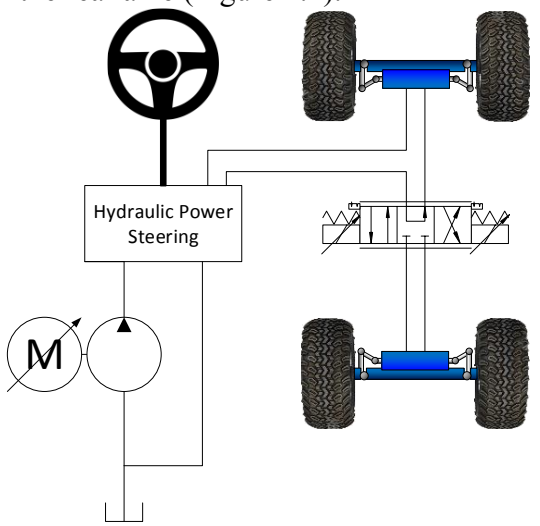

Figure 1.1: Typical hydraulic steering system layout

The series connection, where the front cylinder outlet port is connected to the rear cylinder inlet port, halves the total available force on the front axle cylinder during the steering maneuvers due to the rear cylinder force requirement. Consequently, the vehicle loses its steering full capability in terms of maximum achievable angle. Furthermore, switching from $2 \mathrm{WS}$ to $4 \mathrm{WS}$ mode, and vice versa, it is extremely uncomfortable and time consuming due to the manually alignment of all the wheels by the operator. In addition, this type of steering system forces at least one of the wheels to slip during the machine maneuvers due to the geometrical Ackermann error (being the kinematics typically not Ackermann) unless using complex kinematics [1] thus increasing fuel and tires consumption.

Steering systems have been studying with different objectives, including energy efficiency, safety, comfort and autonomous vehicles[2], [3], [4].

On the market some electro-hydraulic steering system are already available, among which the ones produced by Mobil Elektronik GmbH and HYDAC.

The first competitor provides an electronic hydraulic rear power steering systems (EHLA), Figure 1.2, [5]. 
In this solution the real axle steering system is of hydraulic-mechanical types and managed and controlled independently from the front axle.

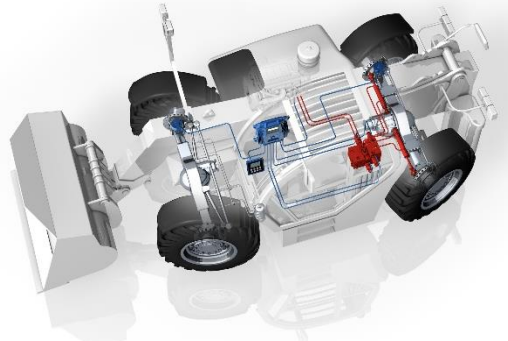

Figure 1.2: Mobil Elektronik EHLA Plus

Different steering modes are provided, such as 4wheel steering, crab steering and manual steering of the rear axle. Moreover, is implemented a smart steering mode "automatic rear swing-out suppression" that prevents the swinging out of the rear part of the vehicle, during steering maneuvers, by turning the rear axle later than the front one.

The second competitor provides a steering system able to steer independently more than two axles [6], i.e. vehicles equipped with multiple axles. Figure 1.5 reports how any single steering axle could be controlled.

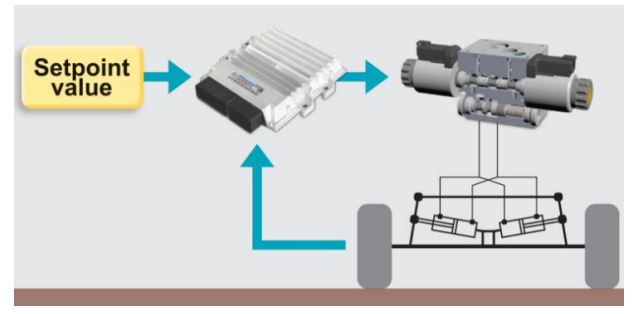

Figure 1.5: HYDAC Steering Architecture

As for the previous solution, there are no mechanical / hydraulic connections between the axles.

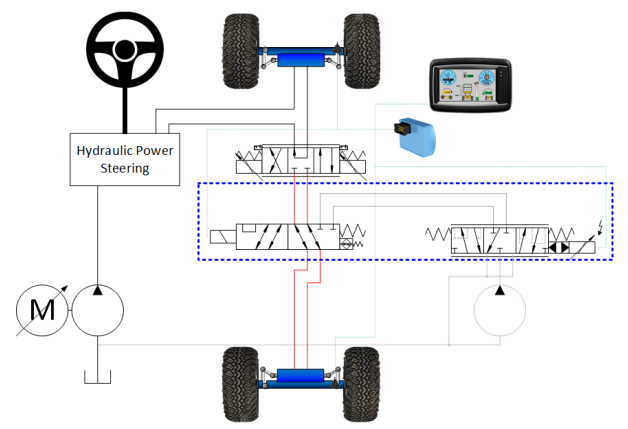

Figure 1.3: iTSS Off
The available steering modes are on-road steering, all-wheel steering and crab steering. In the first a central axle is not steered and works as mid-point while the axles behind it are controlled in function of the front axles. In the second all the axles can be steered, while in the last all the axles are steered equally at the same steering angle resulting in the vehicle sideways movement.

Both the systems include a Fail-Safe Reaction. When an error is detected, the faulty axle is locked into a default position. In this condition the operator will be able to turn the vehicle, but the controllability results compromised.

\section{DANA SOLUTION DESCRIPTION}

The Dana "Intelligent Twin Steering System" (iTSS) is a novel steering system for mobile offhighway vehicles that introduces the possibility to switch from the series (Figure 1.3) to the parallel (Figure 1.4) connection of the hydraulic steering cylinders, advanced steering functions and application-based steering profiles.

The proposed solution adds to the actual steering system (Figure 1.1) a dedicated rear steering directional flow control valve and its controlling electronics.

The valve allows to switch from the series architecture (conventional one) to the parallel architecture, still maintaining the safety.

The iTSS system (Figure 2.1) is composed of:

- an intelligent valve, called iTSS valve;

- angle sensors, to detect front and rear axles steering angles;

- an electronic control unit (ECU) to manage the whole steering system.

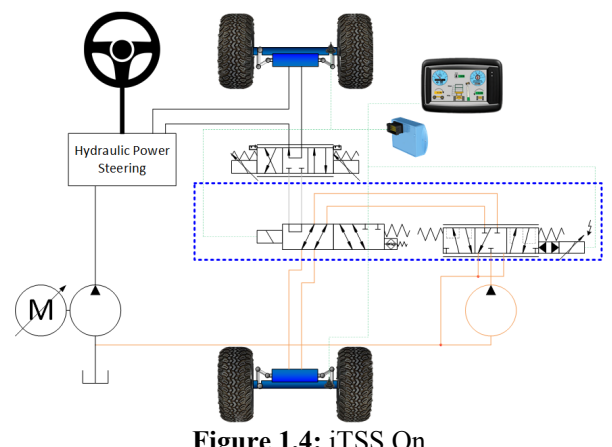

Figure 1.4: iTSS On 


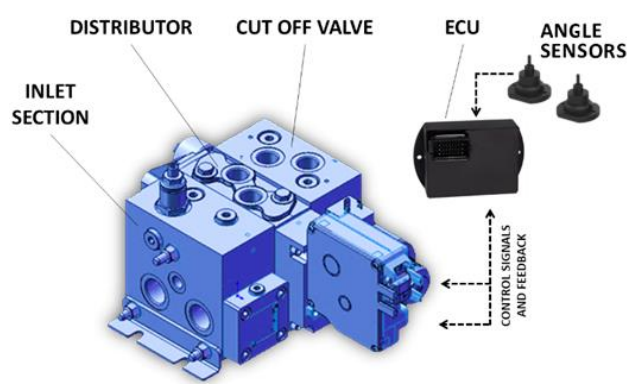

Figure 2.1: iTSS System

The main component of the proposed system is the iTSS valve. This valve is composed of three blocks: The Cut Off Valve, the Inlet Section and the Distributor. When the Cut Off Valve is energized the steering system switches from the traditional series hydraulic architecture to the parallel independent hydraulic architecture, i.e. the real axle is not anymore coupled to the front one but is managed by the electronics through the Distributor block according to the desired strategy. This configuration allows to double the steering force on both the axles (the hydraulic force is not split anymore between the front and rear axles), enable advanced steering functions (described in detail in section 4), and still maintain the safety of the system through dedicated Fail Operational Reaction. The Fail Operational reaction is one of the smartest features of this system. In fact, in case of any dangerous failure, if activated, the Cut Off Valve will be switched off in order to set the steering system from the parallel configuration back to the "conventional" series one. This allows to maintain the traditional steering modes and the control on all the wheels but with lower effectiveness and functionality. This feature is not actually provided by any competitor with similar steering system.

The distributor is the Dana Brevini HPV series 41 , a proportional flow control valve acted by an electronic actuator (Sonceboz Sense 42). The Inlet Block regulates the inlet pressure according to the Load Sensing logic.

The iTSS requires angular (or position) sensors in order to measure the frontal steering angle and controls the rear axles steering angle according to the selected function. The sensors are based on contact-less Hall effect technology. Safety is provided by means of a doubled output signal, one redundant and opposite to the other.
The ECU, developed and programmed by DANA, reads the analog and digital inputs and manages the outputs in order to manage the steering system.

\section{VEHICLE KINEMATICS ANALYSIS AND MATHEMATICAL MODEL}

In this section are reported the kinematics analysis of the proposed steering system and its mathematical model. The aim of the model is that of calculates the wheels paths during arbitrary steering maneuvers and their slipping in order to evaluate and compare different solutions and control strategies.

The simplified scheme of the steering mechanism, installed on all the steering axles produced by Dana, is reported in Figure 3.1, where the neutral position (dashed lines) and a generical position assumed by the mechanism during a steering maneuvers (solid lines) are pointed out in order to identify the variables of interest. Solving the kinematic chain equations it is possible to define the correlation between the steering piston position and the wheels steering angles (Eq.s (3.1), (3.2)).

Knowing the wheels steering angles the position of the front and rear axles instantaneous rotational centers, OF and OR (Figure 3.2), can be calculated (Eq.s (3.3), (3.4)), under the hypothesis of null slip angles, due to the low vehicle speed.

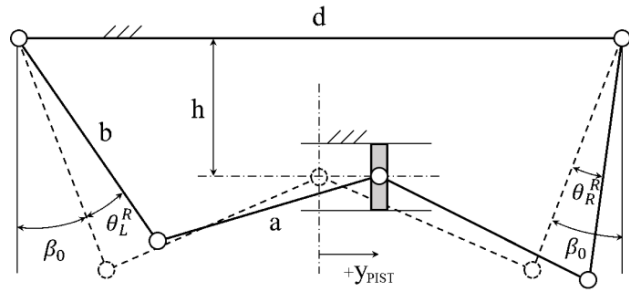

Figure 3.1: Axle simplified steering mechanism scheme

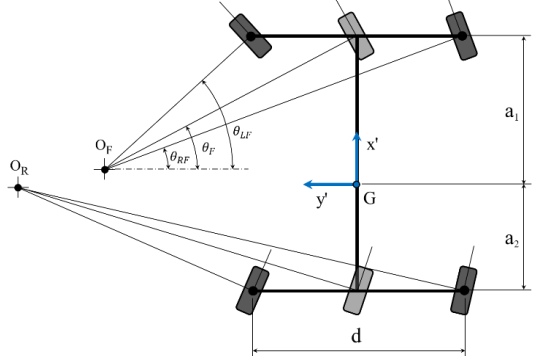

Figure 3.2: Axles rotational centres 


$$
\begin{aligned}
& \theta_{L}{ }^{R}=\frac{\pi}{2}-2 \tan ^{-1}\left(\frac{2 h b+\sqrt{(2 h b)^{2}-\left(\left(\frac{d}{2}+y_{\text {pist }}\right)^{2}+b^{2}+h^{2}-a^{2}\right)^{2}+\left(2\left(\frac{d}{2}+y_{\text {pist }}\right) b\right)^{2}}}{\left(\frac{d}{2}+y_{\text {pist }}\right)^{2}+b^{2}+h^{2}-a^{2}+2\left(\frac{d}{2}+y_{\text {pist }}\right) b}\right) \\
& \theta_{R}{ }^{R}=2 \tan ^{-1}\left(\frac{2 h b+\sqrt{(2 h b)^{2}-\left(\left(\frac{d}{2}-y_{p i s t}\right)^{2}+b^{2}+h^{2}-a^{2}\right)^{2}+\left(2\left(\frac{d}{2}-y_{p i s t}\right) b\right)^{2}}}{\left(\frac{d}{2}-y_{\text {pist }}\right)^{2}+b^{2}+h^{2}-a^{2}+2\left(\frac{d}{2}-y_{\text {pist }}\right) b}\right)+\beta_{0} \\
& -\frac{\pi}{2} \\
& \left\{\begin{array}{c}
x_{O_{F}}=a_{1}+\frac{d}{2} \tan \theta_{L F}+\frac{d}{2}\left(\frac{\tan \theta_{R F}+\tan \theta_{L F}}{\tan \theta_{R F}-\tan \theta_{L F}}\right) \tan \theta_{L F} \\
y_{O_{F}}=-\frac{d}{2}\left(\frac{\tan \theta_{R F}+\tan \theta_{L F}}{\tan \theta_{R F}-\tan \theta_{L F}}\right)
\end{array}\right. \\
& \left\{\begin{array}{c}
x_{O_{R}}=-a_{2}+\frac{d}{2} \tan \theta_{L R}-\frac{d}{2}\left(\frac{\tan \theta_{L R}+\tan \theta_{R R}}{\tan \theta_{L R}-\tan \theta_{R R}}\right) \tan \theta_{L R} \\
y_{O_{R}}=\frac{d}{2}\left(\frac{\tan \theta_{L R}+\tan \theta_{R R}}{\tan \theta_{L R} \tan \theta_{R R}}\right)
\end{array}\right.
\end{aligned}
$$

In order to define the vehicle instantaneous rotational center $\left(\mathrm{O}^{\prime}\right)$ the equivalent bicycle model has been considered (Figure 3.3).

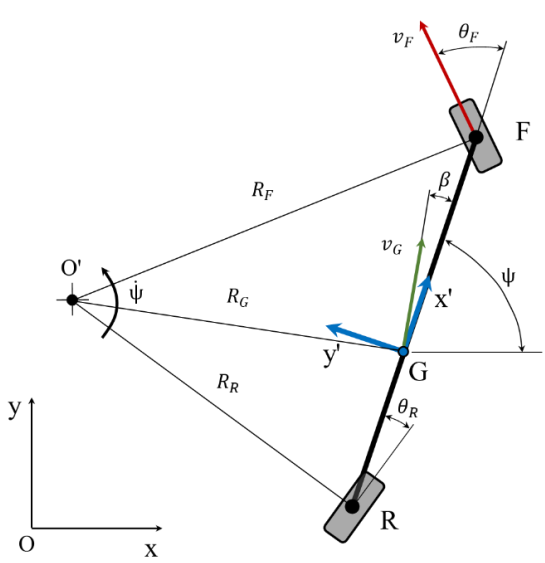

Figure 3.3: Equivalent bicycle model

Eq. (3.5) relates the wheels steering angles with the central axle equivalent wheel steering angle. This equation can be adopted for both front and rear axles.

$$
\tan \theta_{F}=\frac{2 \tan \theta_{L F} \tan \theta_{R F}}{\tan \theta_{L F}+\tan \theta_{R F}}
$$

Following the approach reported in [7] the vehicle slip angle (Eq. (3.6)) and equations describing the equivalent bicycle model center of mass velocity in the plane (Eq.s (3.7)).

$$
\begin{gathered}
\beta=\tan ^{-1}\left(\frac{a_{1} \tan \theta_{R}+a_{2} \tan \theta_{F}}{a_{1}+a_{2}}\right) \\
\left\{\begin{array}{c}
\dot{x_{G}}=v_{G} \cos (\psi+\beta) \\
\dot{y_{G}}=v_{G} \sin (\psi+\beta) \\
\dot{\psi}=\frac{v_{G} \cos \beta\left(\tan \theta_{F}-\tan \theta_{R}\right)}{L}
\end{array}\right.
\end{gathered}
$$

Once integrated in time the vehicle inclination and center of mass position can be found as reported in Eq.s (3.8).

$$
\begin{gathered}
\psi\left(t_{i}\right)=\psi\left(t_{i-1}\right)+\dot{\psi}_{i} \Delta t \\
x_{G}\left(t_{i}\right)=x_{G}\left(t_{i-1}\right)+v_{G_{i}} \cos \left(\psi\left(t_{i}\right)+\beta_{i}\right) \Delta t \\
y_{G}\left(t_{i}\right)=y_{G}\left(t_{i-1}\right)+v_{G_{i}} \sin \left(\psi\left(t_{i}\right)+\beta_{i}\right) \Delta t
\end{gathered}
$$

Where

$$
\dot{\psi}_{i}=\frac{v_{G}}{R_{G_{i}}}
$$

and

$$
R_{G_{i}}=\frac{a_{1}+a_{2}}{\cos \beta_{i}\left(\tan \theta_{F_{i}}-\tan \theta_{R_{i}}\right)}
$$

Defining a fixed reference system (Oxy) and knowing the vehicle center of mass position and inclination, referring to Figure 3.4, it is now possible to evaluate the position of the wheels (A, $\mathrm{B}, \mathrm{C}, \mathrm{D})$. The calculation of the wheel A has been 


$$
\left.(A-O)\right|_{o x y}=\left(\begin{array}{c}
x_{G}+\left(a_{1}-e \sin \theta_{L F}\right) \cos \psi-\left(\frac{w}{2}+e \cos \theta_{L F}\right) \sin \psi \\
y_{G}+\left(a_{1}-e \sin \theta_{L F}\right) \sin \psi+\left(\frac{w}{2}+e \cos \theta_{L F}\right) \cos \psi \\
0
\end{array}\right)
$$

following reported (Eq.s (3.11), (3.13)). This approach can be adopted for all the wheels.

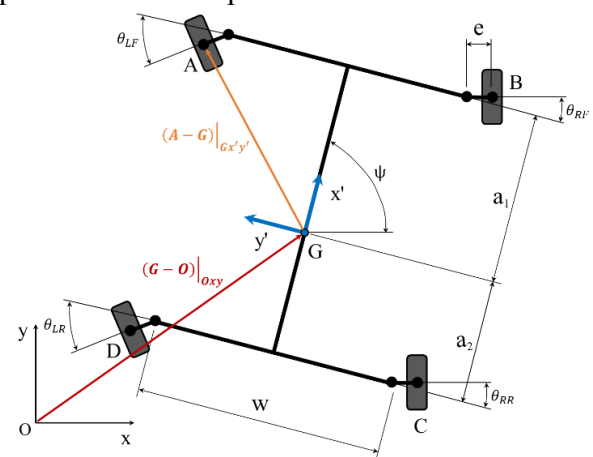

Figure 3.4: Wheels position calculation

$$
\begin{aligned}
\left.(A-O)\right|_{o x y}= & \left.(G-O)\right|_{O x y} \\
& +\left.\boldsymbol{R}(A-G)\right|_{G x \prime y}
\end{aligned}
$$

Where $\mathbf{R}$ (Eq. (3.12)) represents the rotational matrix of the vehicle referring to the fixed reference system.

$$
\mathbf{R}=\left[\begin{array}{ccc}
\cos \psi & -\sin \psi & 0 \\
\sin \psi & \cos \psi & 0 \\
0 & 0 & 1
\end{array}\right]
$$

\section{ADVANCED FUNCTIONS}

The reported steering functions take advantage of the independency of the front and rear axles in order to implement special steering manoeuvres.

\subsection{Follow the path}

This advanced steering function has been defined in order to overlap the rear wheels steering traces to the front wheel ones.

When the front axle starts steering, the action on the rear axle is then properly delayed.

The setpoint at "Regime" of the rear axle is calculated by Eq.(3.16).

$$
\theta_{R}^{\text {Regime }}=-\theta_{F}
$$

From the setpoint, the control system works as in Figure 3.5:

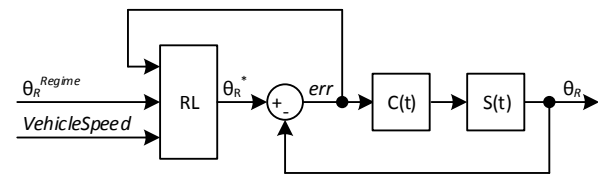

Figure 3.5: Follow the Path Control System

The setpoint of the rear axle $\theta_{R}^{\text {Regime }}$ is reached by a dynamic Rate Limiter $\left(R L_{D y n}\right)$, which delays the reaching of it.

$$
\begin{aligned}
& R L_{B}=\frac{(\text { VehicleSpeed })}{3,6 \times \text { AxlesDistance }} \times \Delta t \\
& R L_{D y n}=R L_{B} \times \max \left(\left|\theta_{R}-\theta_{R}{ }^{\text {Regime }}\right| \times K_{1} ; K_{2}\right)
\end{aligned}
$$

Where $K_{1}$ and $K_{2}$ are parameters tuned for the specific vehicle / application.

Considering:

$$
\text { err }=\theta_{R}^{*}-\theta_{R}
$$

The value of $R L_{D y n}$ is the maximum quantity (in degrees) to be applied to increment / decrement the reference: $\theta_{R}{ }^{*}$ in $\Delta \mathrm{t}$ time in order to reach the $\theta_{R}{ }^{R e g}$. The Formula is detailed in Eq. (4.1).

This function is very useful in both on-road and on-field working conditions, because it allows to easily avoid obstacles and reducing the crop damaged by the wheels path during headland turning sequence.

\subsection{Kinematronic Rotation Centre}

During steering maneuvers due to the geometrical configuration of the axles is typically generated an Ackermann error (Figure 3.6). The effect of this error is that slippage occurs on the

$$
\theta_{R}{ }^{*}(t)=\left\{\begin{array}{cl}
\theta_{R}{ }^{R e g} & |e r r| \leq R L_{D y n} \\
\theta_{R}(t-\Delta t)+\operatorname{sign}(\text { err }) \times R L_{D y n} & |e r r|>R L_{D y n}
\end{array}\right.
$$


wheels during the steering maneuver. Thus, increasing tires and fuel consumption.

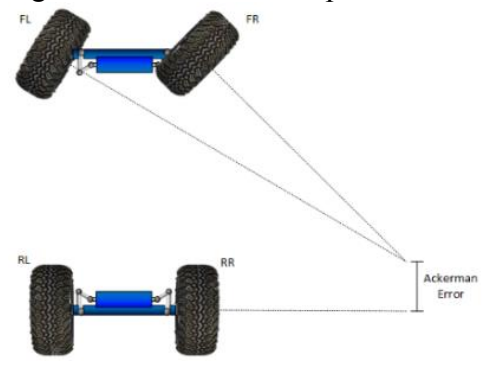

Figure 3.6: Ackermann Error

Ideal steering trajectories, i.e. with no Ackerman error, occur when all the wheels angle have a singular vehicle rotational center. Thanks to the capability of iTSS to independently control the rear axle steering angle from the front one, it is possible to minimize the Ackerman error for any front steering angle. The Kinematronic idea is to overlap the rear axle rotational center on the front axle one (Figure 3.7), thus the rear steering angle will be calculated by the ECU as a function of the front steering angle.

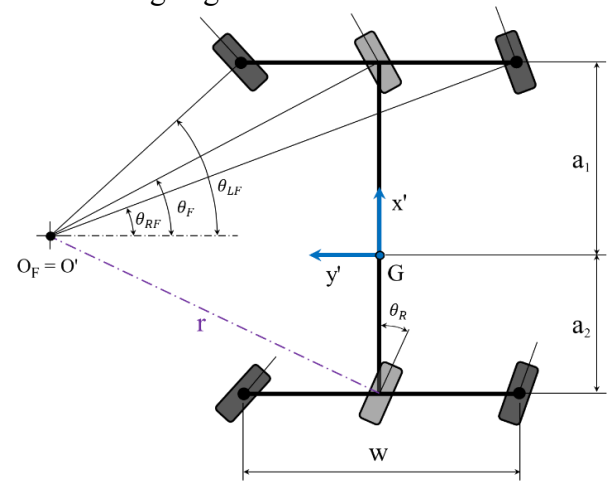

Figure 3.7: Kinematronic conceptual idea

\section{RESULTS}

In this section the wheels trajectories have been reported and compared between traditional steering configuration and the presented (Section 4) advance steering function during typical steering maneuvers. The calculation took count of the vehicle and axles geometrical dimensions, front and rear axle steering angles and vehicle speed.

The colour codes of the wheels paths on the plane is represented in Figure 5.1.

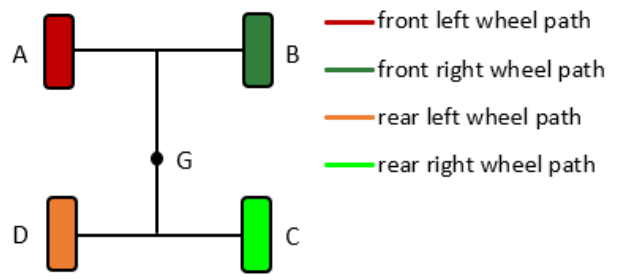

Figure 5.1: Wheels colour codes

\subsection{Follow the path vs 2 WS}

Figure 5.2 reports the wheels path during a generic $\mathrm{S}$ steering maneuver of the considered vehicle in the $2 \mathrm{WS}$ mode, while Figure 5.3 depicts the same steering maneuver with the 4WS steering mode and the "Follow the path" enabled. Comparing the trajectories, it is possible to point out that by means of Follow the Path function the steering maneuvers results more effective in term of impacted area. Thus, it becomes easier and safer to avoid obstacles and turn with long vehicles and vehicles with trailer.

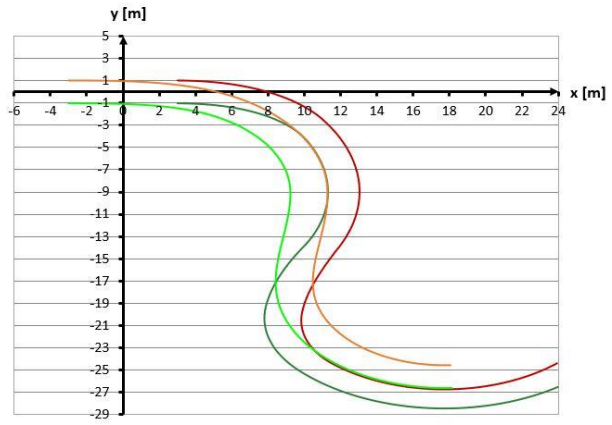

Figure 5.2: Wheels trajectory in 2WS steering configuration

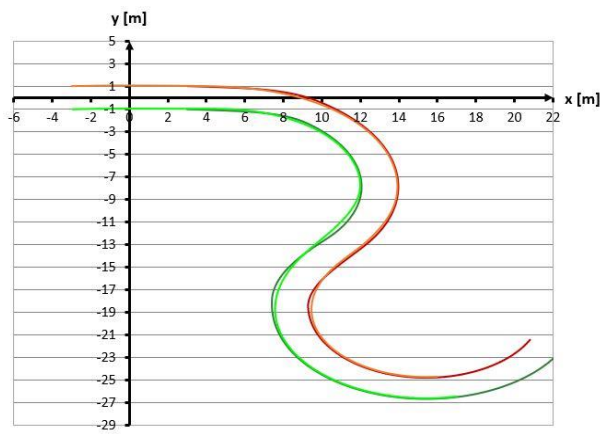

Figure 5.3: Wheels trajectory in 4WS and Follow the Path mode 


\subsection{Follow the path vs $4 \mathrm{WS}$ (Async)}

Figure 5.4 reports the wheels path of sprayers, tractors or any other agricultural machine during a headland turning sequence of the considered vehicle in the 4WS asynchronous mode, while Figure 5.5 depicts the same steering maneuver with the 4WS steering mode and the "Follow the path" enabled.

In the first steering mode this maneuver cause much more crop damaging due to the four different wheels path on the ground compared to the Follow the Path mode that left only two traces on the ground.

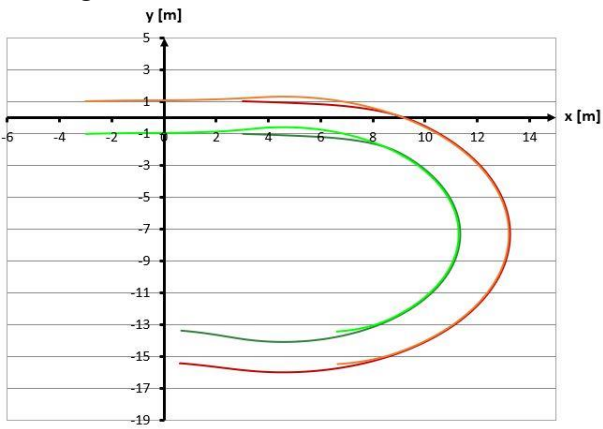

Figure 5.4: Vehicle Trajectory with Follow the Path NOT Active (4WS Async Active)

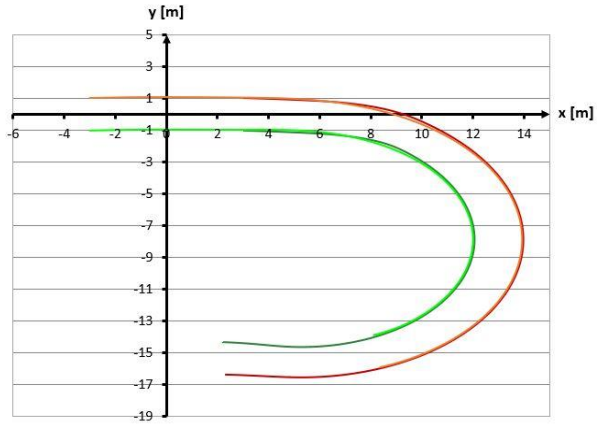

Figure 5.5: Vehicle Trajectory with Follow the Path Active

\subsection{Kinematronic Rotation Centre vs 2WS}

The generic $\mathrm{S}$ steering manoeuvre reported in Figure 5.2 has been performed with the conventional 2WS steering mode and the Kinematronic mode.

As reported in section 4, the Kinematronic algorithm works with the aim to reduce the Ackermann error controlling the rear axle steering angle (Figure 3.7). Due to the steering mechanism geometry the position of the real rear rotation centre is not the same of the calculated one (Figure 5.6). This introduces a slip velocity $\left(v_{L R, S}\right)$, as depicted in Figure 5.7 for the rear left wheel only for simplicity, normal to the rolling velocity $\left(v_{L R}\right)$.

The slippage on the front wheels has been neglected, while the total rear wheels slippage has been calculated integrating the slippage speed $\left(\left|v_{L R, S}\right|\right)$ in time.

Nevertheless, thanks to the Kinematronic it is possible to reduce the wheels slippage up to $86 \%$ in the considered manoeuvre and similar improvements can be obtained for any other steering movement.

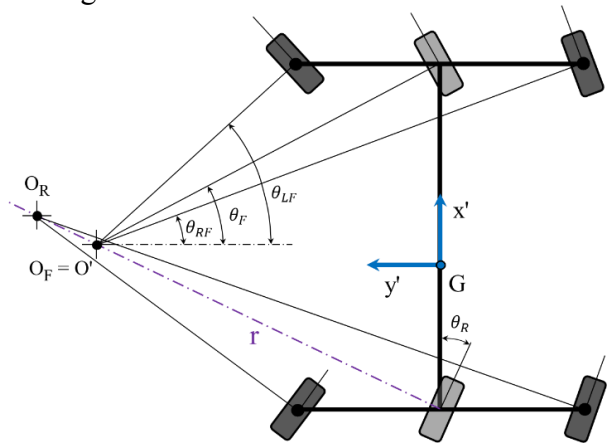

Figure 5.6: Vehicle rotation centre with kinematronic enabled

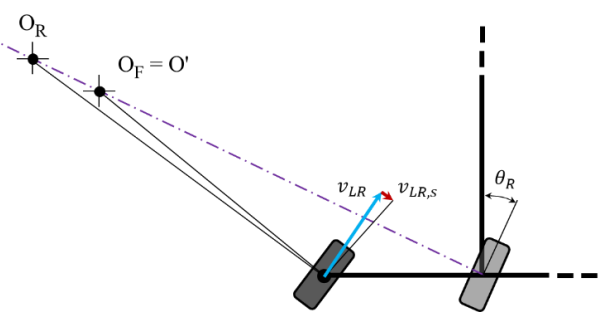

Figure 5.7: Left rear wheel velocities

\section{CONCLUSION AND OUTLOOK}

In this contribution, a novel electro-hydraulic steering system for mobile vehicles and dedicated advanced functions have been presented and described in detail. The proposed iTSS system introduces significant improvements in terms of functionality, comfort and performance compared to traditional steering configurations. Moreover, the DANA solution differs from other similar solutions already available on the market for the available steering functions and safety features. 


\section{NOMENCLATURE}

a Steering mechanism crank length [m]

$a_{1} \quad$ Distance between centre of mass and front axle [m]

$a_{2} \quad$ Distance between centre of mass and rear axle [m]

$b \quad$ Steering mechanism outrigger length [m]

$d \quad$ Distance between wheels rotational centres [m]

$e \quad$ Wheels link length [m]

err Error value in follow the path control $\left[^{\circ}\right]$

$G \quad$ Vehicle centre of mass

$h \quad$ Distance between wheels axle and the steering hydraulic actuator $[\mathrm{m}]$

$O_{F} \quad$ Front axle rotational centre

$O_{R} \quad$ Rear axle rotational centre

$O^{\prime} \quad$ Equivalent bicycle rotational centre

$R_{F} \quad$ Front axle radius [m]

$R_{G} \quad$ Centre of mass radius [m]

$R_{R} \quad$ Rear axle radius [m]

$R L_{B} \quad$ Rate Limiter Base Value for follow the path algorithm, it is used to calculate the $R L_{D y n}$ value

$R L_{D y n}$ Rate Limiter Dynamic Value for follow the path algorithm [rad]

$v_{F} \quad$ Front equivalent wheel velocity $[\mathrm{m} / \mathrm{s}]$

$v_{G} \quad$ Centre of mass velocity $[\mathrm{m} / \mathrm{s}]$

$x_{G} \quad$ Centre of mass x-coordinate [m]

$\dot{x}_{G} \quad$ Centre of mass velocity $\mathrm{x}$-coordinate $[\mathrm{m} / \mathrm{s}]$

$y_{G} \quad$ Centre of mass y-coordinate $[\mathrm{m}]$

$\dot{y}_{G} \quad$ Centre of mass velocity y-coordinate $[\mathrm{m} / \mathrm{s}]$

$y_{P I S T} \quad$ Steering hydraulic cylinder stroke $[\mathrm{m}]$

$\beta \quad$ Vehicle slip angle [rad]

$\beta_{0} \quad$ Initial axle steering angle [rad]

$\psi \quad$ Vehicle inclination angle [rad]

$\dot{\psi} \quad$ Vehicle rotational speed [ $\mathrm{rad} / \mathrm{s}]$

$\theta_{R}^{R e g}$ Value to be reached on rear axle at regime when follow the path algorithm is working [rad]

$\theta_{F} \quad$ Front wheel steering angle [rad]

$\theta_{L F} \quad$ Front axle left wheel steering angle [rad]

$\theta_{L R} \quad$ Rear axle left wheel steering angle [rad]

$\theta_{R} \quad$ Rear wheel steering angle [rad]

$\theta_{R F} \quad$ Front axle right wheel steering angle [rad]

$\theta_{R R} \quad$ Rear axle right wheel steering angle [rad]

\section{REFERENCES}

[1] Jing-Shan Zhao, Jian S Dai. Design of an Ackermann Type Steering Mechanism. Proceedings of the Institution of Mechanical Engineers Part C Journal of Mechanical Engineering Science 1989-1996 (vols 203-210), November 2013.2 DOI: $10.1177 / 0954406213475980$

[2] Hiroyuki Miyazaki. Technical trends in steering systems. Proceedings of the 7th JFPS International Symposium on Fluid Power, TOYAMA 2008. September 15-18, 2008. ISBN 4-931070-07-X

[3] Keiichi Inoue. Steering control system for autonomous tractor. Proceedings of the 7th JFPS International Symposium on Fluid Power, TOYAMA 2008. September 15-18, 2008. ISBN 4-931070-07-X

[4] Arun Singh, Abhishek Kumar, Rajiv Chaudhary, R. C. Singh. Study of 4 Wheel Steering Systems to Reduce Turning Radius and Increase Stability. International Conference of Advance Research and Innovation (ICARI-2014). ISBN 978-935156-328-0

[5] http://www.mobilelektronik.com/en/systems/ehla.html [access date: 01/10/2019]

[6] https:/www.hydac.com/fileadmin/pdb/ pdf/PRO0000000000000000000101169000011. pdf [access date: 01/10/2019]

[7] R. Rajamani, Vehicle Dynamics and Control, Springer Science \& Business Media, 2011. ISBN 0-387-26396-9 